\title{
Comparative evaluation of the Sticky-Resting- Box-Trap, the standardised resting-bucket-trap and indoor aspiration for sampling malaria vectors
}

\author{
Katharina S. Kreppel ${ }^{1,2^{*}}$, P. C. D. Johnson ${ }^{1}$, N. J. Govella ${ }^{2}$, M. Pombi ${ }^{3}$, D. Maliti ${ }^{1,2}$ and H. M. Ferguson ${ }^{1}$
}

\begin{abstract}
Background: Understanding mosquito resting behaviour is important for the control of vector-borne diseases, but this remains a challenge because of the paucity of efficient sampling tools. We evaluated two novel sampling methods in the field: the Sticky Resting Box (SRB) and the Resting Bucket trap (RBu) to test their efficiency for sampling malaria vectors resting outdoors and inside houses in rural Tanzania. The performance of RBu and SRB was compared outdoors, while indoors SRB were compared with the Back Pack Aspiration method (BP). Trapping was conducted within 4 villages in the Kilombero Valley, Tanzania over 14 nights. On each night, the performance for collecting Anopheles vectors and Culicinae was compared in 4 households by SRB and RBu outdoors and by SRB or fixed-time Back Pack aspirator in 2 of the 4 focal households indoors.
\end{abstract}

Findings: A total of 619 Anopheles gambiae s.l., 224 Anopheles funestus s.l. and 1737 Culicinae mosquitoes were captured. The mean abundance of An. arabiensis and An. funestus s.l. collected with SRB traps inside and outdoors was significantly lower than with BP or RBu. The SRB however, outperformed BP aspiration for collection of Culicinae indoors.

Conclusions: Of the methods trialled indoors (BP and SRB), BP was the most effective, whilst outdoors RBu performed much better than SRB. However, as SRB can passively sample mosquitoes over a week they could provide an alternative to the RBu where daily monitoring is not possible.

Keywords: Anopheles arabiensis, Malaria vector, Resting behaviour, Exophily, Sticky trap, Ecology, Resting traps

\section{Findings}

Currently there are few reliable and widely applicable methods for studying the resting behaviour of Anopheles malaria vectors in outdoor and indoor settings. Conventional methods of collecting mosquitoes resting indoors include Pyrethrum Spray Catches (PSC) and active mouth/electric aspirations [1]. Current tools for sampling mosquitoes outdoors include Muirhead-Thomson PitShelters [2], resting boxes [3, 4] or clay pots [5]. No standardised method for use indoors and outdoors is available, making it difficult to infer unbiased estimates of mosquito resting habitat preference from these current methods.

\footnotetext{
* Correspondence: Katharina.Kreppel@glasgow.ac.uk

'Institute of Biodiversity, Animal Health and Comparative Medicine, University of Glasgow, Glasgow, UK

${ }^{2}$ Environmental Health and Ecological Sciences group, Ifakara Health Institute, Dar-es-Salaam, Tanzania

Full list of author information is available at the end of the article
}

This study investigated the performance of two lightweight, portable traps for collecting resting mosquitoes in rural Tanzania: the recently developed Sticky Resting Box (SRB) [6], a variant of the mosquito Resting Box [4], and the Resting Bucket ( $\mathrm{RBu})$, a new trap presented here. Both traps are inexpensive and easy to produce from materials readily available in rural African settings. These features would make it easy to deploy these traps in high numbers as part of routine vector surveillance.

\section{Methods}

Mosquitoes were collected within 4 villages in the Kilombero Valley in south-eastern Tanzania: Kidugalo (S08 ${ }^{\circ}$ 30.7258'; E036 $31.8476^{\prime}$ ), Lupiro (S08 $23.2956^{\prime}$; E036 40.6122'), Minepa (S08 $16.4974^{\prime}$; E036 $\left.40.7640^{\prime}\right)$ and Sagamaganga (S08 $03.8392^{\prime}$; E036 $47.7709^{\prime}$ ) where malaria is endemic. The dominant malaria vectors are $A n$. 
arabiensis and An. funestus s.l. Trapping of resting mosquitoes was conducted indoors and outdoors at 4 households in each village over 14 nights between August and September 2012, coincident with the end of the dry season. In each village, the owner of a randomly selected house based on a 2012 IHI DSS census list and owners of 3 neighbouring houses (100-200 m distance) were asked to partake.

The SRB trap is - as described in Pombi et al. [6] - a rectangular wooden box, lined with black cotton cloth inside on top of which glue covered acetate sheets are attached to trap mosquitoes that enter to rest (Fig. 1a and b). During trapping it was placed on its side, and partially opened on one end to allow mosquitoes to enter. The other outdoor trap used was the RBu (Fig. 1c) made from a standard 201 plastic bucket $(290 \mathrm{~mm}$ diameter opening and $390 \mathrm{~mm}$ deep) also lined with black cotton fabric, and set by placing it on its side with the opening facing a house. Inside both traps a wet cloth was placed to increase humidity. Within the desired $5 \mathrm{~m}$ distance range from households, outdoor traps were positioned facing the house in relatively shady areas, ideally next to or under vegetation. Each night two SRBs and three RBus per household per village were set up outdoors. SRB traps were also used to trap mosquitoes resting indoors, with one trap being placed on the floor in the sleeping room or its entrance area. Indoor resting mosquitoes were also collected by sweeping a CDC Back Pack aspirator (BP, Model 1412, John Hock, Florida USA, Fig. 1d [7]) over the walls and ceiling of sleeping rooms for a 10 min period. All SRB and RBu traps were set at dusk. All SRB and RBu were checked and indoor BP aspiration conducted between 6.00-8.00 am the next morning. Mosquitoes resting inside RBus were collected using a CDC backpack aspirator and aspirating for 10-20 $\mathrm{s}$ at the open end of the bucket. Whilst only the mosquitoes resting inside $\mathrm{RBu}$ and $\mathrm{SRB}$ or on walls at the time of sampling would be collected, the sticky lining of the SRB meant that any mosquito resting in the trap during the preceding $12 \mathrm{~h}$ should have been caught.

All trap types were tested on all nights of the study. However, the two indoor collections per house per night (BP and SRB) could not be compared simultaneously in the same house because of their potential competition for mosquitoes within the same room. Consequently a modified Latin-square design was used in which only one indoor trapping method was used per house per day. On each night, one SRB was placed in a sleeping room of 2 houses, whilst BP aspiration was conducted in the remaining two. Over successive nights, trapping methods and collectors were rotated through households.

All mosquitoes caught were morphologically identified to genera [8] and a subset of those identified as belonging to the An. gambiae s.l. species group $(n=285,46 \%$ of the

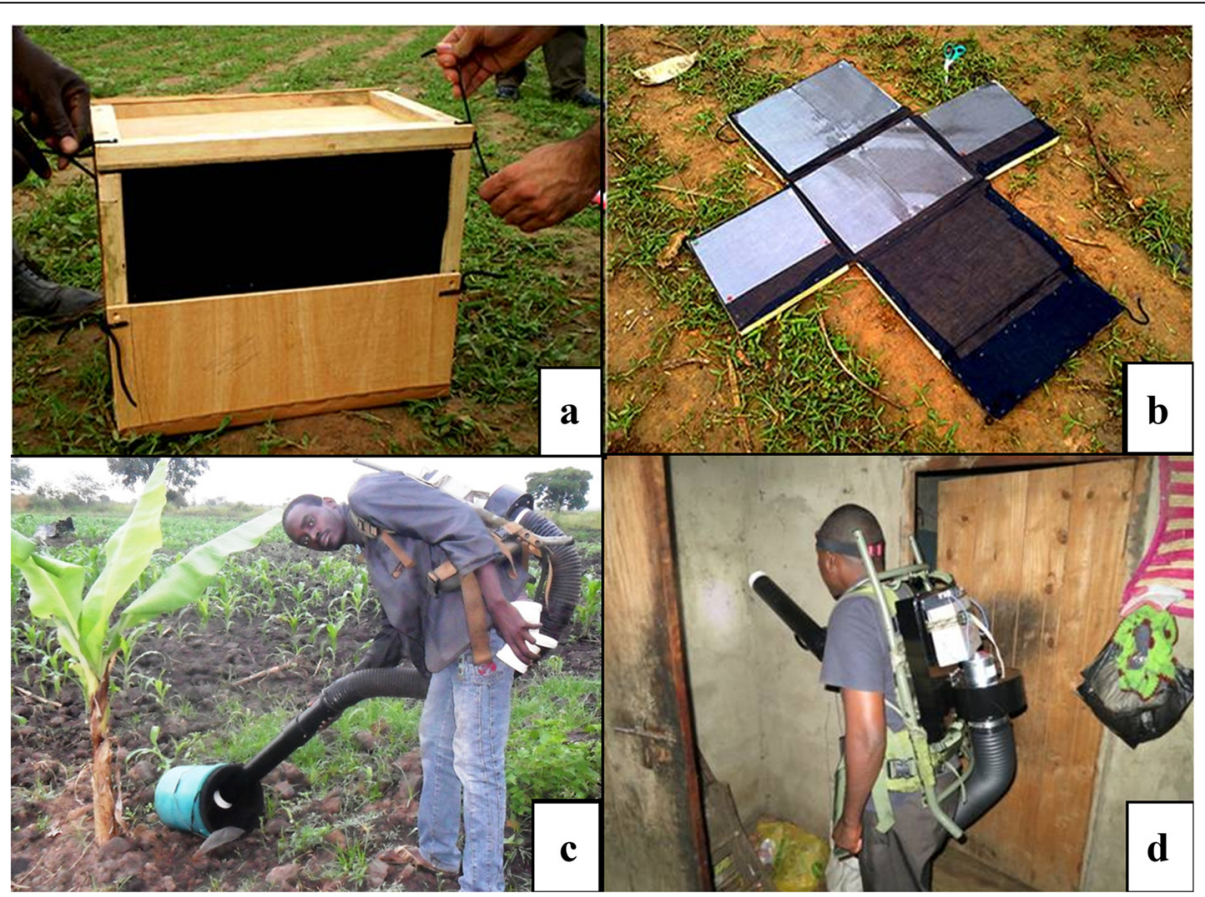

Fig. 1 Trapping methods to collect resting mosquitoes a) Sticky Resting Box trap (30 cm $\times 60 \mathrm{~cm} \times 30 \mathrm{~cm}$ ) fully assembled, lined with black cloth b) Sticky Resting Box trap opened with six A4 acetate sheets covered in glue c) Resting Bucket trap made from a standard $20 \mathrm{I}$ plastic bucket, lined with black cloth, aspirated by CDC backpack aspirator d) Standard battery-powered CDC Back pack aspirator (Model 1412, John Hock, Florida USA) 
Table 1 Total counts of mosquitoes collected by species per trap type regardless of number of traps

\begin{tabular}{llllll}
\hline Mosquito type & \multicolumn{2}{l}{ Outdoors } & & \multicolumn{2}{l}{ Indoors } \\
\cline { 3 - 4 } & RBu & SRB & & BP & SRB \\
\hline An. arabiensis & 514 & 17 & 83 & 5 \\
An. funestus s.l. & 152 & 3 & 69 & 0 \\
Culicinae & 443 & 490 & 734 & 70 \\
Total & 1109 & 510 & 886 & 75 \\
\hline
\end{tabular}

total) were identified to species level by PCR [9]. Before the study began, permission of community leaders in all four villages was sought and informed consent obtained from each head of household where trapping was conducted. Ethical approval and research clearance was obtained from the institutional review board of Ifakara Health Institute in Tanzania (IHI/IRB/No. A50) and the Medical Research Coordination Committee of the National Institute of Medical Research in Tanzania (NIMR/HQ/ R.8c/Vol.ii/125).

Statistical analysis was conducted to compare the mean abundance of malaria vectors and Culicinae captured per night by each trapping method using the statistical software $\mathrm{R}$ [10]. Separate analyses were done for mosquitoes caught indoors (SRB vs BP) and outdoors (SRB vs RBu). Variation in the proportion of malaria vectors (combining An. gambiae s.l. and $A n$. funestus s.l.) within the total mosquito catch was investigated. Generalised linear mixed effects models (GLMM, package lme4 in R) were used, fitting trap type as fixed effect, and night, date and house as random effects.

\section{Results and discussion}

Forty six percent of the An. gambiae s.l. sample were molecularly analysed and all were identified as An. arabiensis. As this finding matches other observations indicating that $>93 \%$ of An. gambiae s.l. from the Kilombero Valley are An. arabiensis [11], it was assumed that all An. gambiae s.l. caught in this study represent $A n$. arabiensis.

In total, 619 An. gambiae s.l. and 1737 Culicinae mosquitoes were captured (Table 1). All traps predicted similar geographical trends in mosquito abundance with numbers estimated as being highest in Lupiro, followed by Minepa and Kidugalo and lastly Sagamaganga.

Outdoors, the $\mathrm{RBu}$ collected significantly more malaria vector species per trap per night than the SRB (Table 2, >20-35 more An. arabiensis and An. funestus s.l., respectively) while the SRB caught approximately 1.25 times more Culicinae than the $\mathrm{RBu}$ (Table 1). Controlling for random variation between villages, the $\mathrm{RBu}$ was estimated to be significantly more efficient at sampling $A n$. arabiensis $\left(\chi^{2}=15.01, p<0.001\right)$ and An. funestus s.l. $\left(\chi^{2}=13.3, p\right.$ $=0.002)$ than the SRB, which in turn collected significantly more Culicinae than the $\mathrm{RBu}\left(\chi^{2}=4.99, p=0.02\right.$; Table 2).

Indoors, the SRB was much less efficient in sampling $A n$. arabiensis than the Back Pack Aspiration (BP), and failed to capture a single An. funestus s.l. (Table 1). Regardless of trap type, malaria vectors constituted a minority of indoorresting mosquitoes (17 \% or less, Table 1$)$. The proportion of vectors estimated caught in BP collections was $\sim 6$ times higher than in SRB.

The BP caught approximately 20 times more $A n$. arabiensis per collection than the SRB, 1.7 times more An. funestus s.l. and 24 times more Culicinae (Table 3), with significant differences for An. arabiensis $\left(\chi^{2}=5.01\right.$, $p=0.02)$, An. funestus s.l. $\left(\chi^{2}=6.73, p=0.009\right)$ and Culicinae $\left(\chi^{2}=6.37, p=0.01\right)$.

Our finding that $A n$. arabiensis was caught resting mainly outdoors is in line with its known exophilic behaviour [12-14], as was the observation that the more endophilic An. funestus s.l. $[15,16]$ was primarily caught indoors. However, the SRB only caught An. funestus s.l. outdoors, as also found in Burkina Faso [6]. Whilst SRB caught very few malaria vectors, it caught a much higher abundance of Culicinae than either $\mathrm{RBu}$ (outdoors) or $\mathrm{BP}$ (indoors), with the majority being gravid females. This is in agreement with findings from Burkina Faso, where SRB showed high performance in collecting Culicinae [6]. A potential explanation for the attraction of gravid Culicinae to the SRB could be the scent of the glue (polybutylene based adhesives are used in oviposition traps $[17,18])$ which may mimic oviposition odour cues used by these species [19]. Findings such as by Lindh et al. [20] indicate that odour cues are very species specific. This is also supported by results from

Table 2 Predicted mean abundance of mosquito groups per trap per night caught outdoors

\begin{tabular}{|c|c|c|c|c|c|c|}
\hline \multirow[t]{3}{*}{ Village } & \multicolumn{6}{|c|}{ Predicted abundance of mosquitoes per trap per night outdoors } \\
\hline & \multicolumn{2}{|l|}{ An. arabiensis } & \multicolumn{2}{|l|}{ An. funestus s.l. } & \multicolumn{2}{|l|}{ Culicinae } \\
\hline & $\overline{S R B}$ & RBu & $\overline{\mathrm{SRB}}$ & $\mathrm{RBu}$ & $\overline{\mathrm{SRB}}$ & $\mathrm{RBu}$ \\
\hline Kidugalo & * & $0.03(0.004-0.19)$ & * & $0.39(0.15-0.98)$ & $0.0004(40-0.34)$ & $0.0003(0-0.22)$ \\
\hline Lupiro & $0.12(0.03-0.46)$ & $3.71(1.47-9.32)$ & 0 & $1.52(0.49-4.64)$ & $12.11(4.45-32.9)$ & $7.21(2.75-18.84)$ \\
\hline Minepa & $0.39(0.2-0.75)$ & $6.42(4.42-9.31)$ & $0.35(0.16-0.74)$ & $0.68(0.2-2.29)$ & $1.79(0.56-5.68)$ & $0.82(0.27-2.45)$ \\
\hline Sagagmaganga & * & $0.16(0.04-0.66)$ & * & $0.004(0-158.48)$ & $0.000003(0-0.05)$ & $0.00007(0-1)$ \\
\hline Total & $0.03(0.002-0.32)$ & $0.65(0.06-6.69)$ & $0.007(0.002-0.02)$ & $0.25(0.11-0.54)$ & $0.34(0.03-3.05)$ & $0.27(0.03-2.42)$ \\
\hline
\end{tabular}

Numbers in brackets represent $95 \%$ confidence interval. Asterisks indicate where no mosquitoes were caught 
Table 3 Predicted mean abundance of mosquito groups per trap per night caught indoors

\begin{tabular}{|c|c|c|c|c|c|c|}
\hline \multirow[t]{3}{*}{ Village } & \multicolumn{6}{|c|}{ Predicted abundance of mosquitoes per trap per night indoors } \\
\hline & \multicolumn{2}{|l|}{ An. arabiensis } & \multicolumn{2}{|c|}{ An. funestus s.l. } & \multicolumn{2}{|l|}{ Culicinae } \\
\hline & $\overline{S R B}$ & $\mathrm{BP}$ & SRB & $\mathrm{BP}$ & SRB & $\mathrm{BP}$ \\
\hline Kidugalo & * & $0.16(0.02-1.13)$ & * & $1.84(0.8-4.19)$ & * & $1.85(0.7-4.83)$ \\
\hline Lupiro & $0.83(0.22-3.08)$ & $9.33(3.36-25.8)$ & * & $4.3(1.15-15.98)$ & $10.5(4.98-22.11)$ & $52.9(25.61-109.24)$ \\
\hline Minepa & * & $2.8(0.88-8.89)$ & * & $0.56(0.08-3.89)$ & $0.71(0.18-2.74)$ & $21.9(7.59-63.11)$ \\
\hline Sagamaganga & * & $0.12(0.01-0.85)$ & * & $0.32(0.02-4.09)$ & $0.28(0.02-3.79)$ & $8.9(3.54-22.35)$ \\
\hline Total & $0.04(0-0.42)$ & $0.82(0.06-6.31)$ & * & $1.77(0.6-5.2)$ & $0.31(0.05-1.77)$ & $7.52(1.5-37.51)$ \\
\hline
\end{tabular}

Numbers in brackets represent $95 \%$ confidence interval. Asterisks indicate where no mosquitoes were caught

Thailand showing high Culex spp. collections with an oviposition trap for Aedes equipped with glue utilised in the SRB [21, 22]. The performance of the SRB for capturing An. gambiae s.l. indoors was relatively low here $(\sim 4 \%$ relative to the $\mathrm{BP})$ in comparison to what was found in a previous study in Burkina Faso ( 16 \%, [6]). Previous studies of sampling methods for mosquitoes have also shown high spatial variability in relative trap performance [23-26] which highlights the need to evaluate traps in different geographical settings. Potential reasons for the apparent differences in the performance of the SRB in Burkina Faso and Tanzania include differences in species composition within the An. gambiae s.l. complex and study design. While data was collected from two sites over 2 years in Burkina Faso, collections were made over 14 trap nights in Tanzania and fewer traps were used. The SRB was positioned facing the house in both studies, but in Burkina Faso the trap was just centimetres from the wall, while it was set at a distance of $5 \mathrm{~m}$ in Tanzania.

\section{Conclusion}

We conclude that currently in the Kilombero Valley in Tanzania, the most efficient means among those tested of sampling resting malaria vectors is the $\mathrm{RBu}$ and $\mathrm{BP}$ for outdoor and indoor settings respectively, with the SRB performing considerably worse in both resting habitats. In addition to higher relative sampling efficiency, practical advantages of the $\mathrm{RBu}$ are its negligible impact on the environment relative to other methods and being the most convenient trap in terms of transport, set up and maintenance. However, despite lower relative sampling efficiency, the SRB retains unique features that could be advantageous. Specifically, the SRB alone can be used for passive sampling over several days without the need for regular checking, thus increasing its performance in terms of mosquitoes collected without an increase of human effort in the field [6]. Additionally, SRB could be recommended in studies where Culicinae are the primary target.

Within the Kilombero Valley where An. arabiensis is the predominant vector, $\mathrm{RBu}$ traps have shown to be an efficient, low cost sampling method for outdoor resting mosquitoes. We believe this method has strong promise to be further developed into a standardised sampling tool and encourage further investigation of its potential use in a wider range of African settings.

Abbreviations

SRB: Sticky resting box; RBu: Resting Bucket; BP: Back pack aspiration.

\section{Competing interests}

The authors declare that they have no competing interests.

\section{Authors' contributions}

The study was conceived by HMF and KSK. DM participated in its design. The field sampling was coordinated and carried out by KSK. KSK, PCDJ, NJG, MP and HMF analysed the results and drafted the manuscript. All authors contributed to and approved the final manuscript.

\section{Acknowledgements}

We would like to thank Alessandra de la Torre and Gerry F. Killeen for their advice and valuable comments on the manuscript. We are grateful to the laboratory at the University of California Davis and Prof. Greg Lanzaro for their contribution to the testing of samples. We are indebted to the field technicians in Ifakara, Tanzania, Mr. Kilalangongono, Mr. Kihonda, and Mr. Magazini for their help with data collection. Thanks also to S.J. Moore for constructive input during the manuscript writing. We also thank the inhabitants of Kidugalo, Lupiro, Minepa and Sagamaganga for their collaboration during field sampling. Financial support was provided by EU-FP7 grant no: 265660 "Avec Net" and National Institutes of Health grant R01Al085175-03.

\section{Author details}

${ }^{1}$ Institute of Biodiversity, Animal Health and Comparative Medicine, University of Glasgow, Glasgow, UK. Environmental Health and Ecological Sciences group, Ifakara Health Institute, Dar-es-Salaam, Tanzania. ${ }^{3}$ Dipartimento di Sanità Pubblica e Malattie Infettive, Università di Roma "Sapienza", Rome, Italy.

Received: 25 June 2015 Accepted: 1 September 2015

Published online: 17 September 2015

\section{References}

1. WHO. Manual on practical entomology in Malaria part 2: methods and techniques. 1975.

2. Silver JB. Mosquito Ecology - Field Sampling Methods. The Netherlands: Springer; 2008. p. 1498

3. Sikulu M, Govella N, Ogoma S, Mpangile J, Kambi S, Kannady K, et al. Comparative evaluation of the Ifakara tent trap-B, the standardized resting boxes and the human landing catch for sampling malaria vectors and other mosquitoes in urban Dar es Salaam. Tanzania Malaria J. 2009;8:197.

4. Kweka E, Mwang'onde B, Kimaro E, Msangi S, Massenga C, Mahande A. A resting box for outdoor sampling of adult Anopheles arabiensis in rice irrigation schemes of lower Moshi, northern Tanzania. Malaria J. 2009;8:82.

5. Odiere M, Bayoh M, Gimnig J, Vulule J, Irungu L, Walker E. Sampling outdoor, resting Anopheles gambiae and other mosquitoes (Diptera: Culicidae) in western Kenya with clay pots. J Med Entomol. 2007;44:14-22. 
6. Pombi M, Guelbeogo W, Kreppel K, Calzetta M, della Traore A, Sanou A, et al. The Sticky Resting Box, a new tool for studying resting behaviour of Afrotropical malaria vectors. Parasit Vectors. 2014;7(1):247.

7. Clark GG, Seda H, Gubler DJ. Use of the "CDC backpack aspirator" for surveillance of Aedes aegypti in San Juan, Puerto Rico. J Am Mosq Control Assoc. 1994;10(1):119-24

8. Gillies M, Coetzee M. A supplement to anophelinae of Africa south of Sahara (Afro-tropical region). Publication South Africa Institute Med Res. 1987;55:1-143

9. Scott J, Brogdon W, Collins F. Identification of single specimens of the Anopheles gambiae complex by the polymerase chain reaction. AmJTrop Med Hyg. 1993;49(4):520-9.

10. Core-Team RD. R: A language and environment for statistical computing. Vienna, Austria: R Foundation for Statistical Computing; 2011.

11. Mayagaya V, Nkwengulila G, Lyimo I, Kihonda J, Mtambala H, Ngonyani $\mathrm{H}_{\text {, }}$ et al. The impact of livestock on the abundance, resting behaviour and sporozoite rate of malaria vectors in southern Tanzania. Malar J. 2015;14(1):17.

12. Mahande A, Mosha F, Mahande J, Kweka E. Feeding and resting behaviour of malaria vector, Anopheles arabiensis with reference to zooprophylaxis. Malar J. 2007;6:100

13. Fornadel CM, Norris LC, Franco V, Norris DE. Unexpected anthropophily in the potential secondary malaria vectors Anopheles coustani s.l. and Anopheles squamosus in Macha, Zambia. Vector Borne Zoonotic Dis. 2011;11(8):1173-9.

14. White G, Magayuka S, Boreham P. Comparative studies on sibling species of the Anopheles gambiae Giles complex (Diptera: Culicidae): bionomics and vectorial activity of species A and species B at Segera, Tanzania. Bull Entomol Res. 1974;62:215-317.

15. Mendis C, Jacobsen J, Gamage-Mendis A, Bule E, Dgedge M, Thompson R, et al. Anopheles arabiensis and An. funestus are equally important vectors of malaria in Matola coastal suburb of Maputo, southern Mozambique. Med Vet Entomol. 2000;14:171-80.

16. Ogoma SB, Lweitoijera DW, Ngonyani H, Furer B, Russell TL, Mukabana WR et al. Screening Mosquito House Entry Points as a Potential Method for Integrated Control of Endophagic Filariasis, Arbovirus and Malaria Vectors. PLoS Negl Trop Dis. 2010;4(8):e773.

17. Williams CR, Long SA, Russell RC, Ritchie SA. Optimizing ovitrap use for Aedes Aegypti in Cairns, Queensland, Australia: effects of some abiotic factors on field efficacy. In: J Am Mosq Control Assoc, vol. 22. Australia: The American Mosquito Control Association; 2006. p. 635-40.

18. Harris C. A simple and efficent tool for trapping gravid Anopheles at breeding sites. Parasites vectors. 2011;4:125.

19. McCall PJ, Eaton G. Olfactory memory in the mosquito Culex quinquefasciatus. Med Vet Entomol. 2001;15:197-203.

20. Lindh JM, Okal MN, Herrera-Varela M, Borg-Karlson A-K, Torto B, Lindsay SW, et al. Discovery of an oviposition attractant for gravid malaria vectors of the Anopheles gambiae species complex. Malar J. 2015:14(1):119.

21. Facchinelli L, Koenraadt CJM, Fanello C, Kijchalao U, Valerio L, Jones JW, et al. Evaluation of a Sticky Trap for Collecting Aedes (Stegomyia) Adults in a Dengue-endemic Area in Thailand. Am J Trop Med Hyg. 2008;78(6):904-9.

22. Thavara U, Tawatsin A, Chansang C, Kong-ngamsuk W, Paosriwong S, Boon-Long J, et al. Larval occurrence, oviposition behavior and biting activity of potential mosquito vectors of dengue on Samui Island, Thailand. J Vector Ecol. 2001;26:172-80

23. Mathenge E, Misiani G, Oulo D, Irungu L, Ndegwa P, Smith T, et al. Comparative performance of the Mbita trap, CDC light trap and the human landing catch in the sampling of Anopheles arabiensis, An. funestus and culicine species in a rice irrigation in western Kenya. Malar J. 2005:4:7.

24. Laganier R, Randimby F, Rajaonarivelo V, Robert V. Is the Mbita trap a reliable tool for evaluating the density of anopheline vectors in the highlands of Madagascar? Malaria J. 2003;2:42.

25. Braimah N, Drakeley C, Kweka E, Mosha F, Helinski M, Pates H, et al. Tests of bednet traps (Mbita traps) for monitoring mosquito populations and time of biting in Tanzania and possible impact of prolonged ITN use. Int J Trop Insect Sci. 2005:00:1-6.

26. Wong J, Bayoh N, Olang G, Killeen G, Hamel M, Vulule J, et al. Standardizing operational vector sampling techniques for measuring malaria transmission intensity: evaluation of six mosquito collection methods in western Kenya. Malar J. 2013:12(1):143.

\section{Submit your next manuscript to BioMed Central and take full advantage of:}

- Convenient online submission

- Thorough peer review

- No space constraints or color figure charges

- Immediate publication on acceptance

- Inclusion in PubMed, CAS, Scopus and Google Scholar

- Research which is freely available for redistribution

Submit your manuscript at www.biomedcentral.com/submit 